\title{
Today's Midrise Housing in Shanghai
}

\author{
GUO ZHAN, ZHENG CAN, and LIU YANCHUAN \\ Tianjin University, China \\ Faculty Sponsor: Liu Tongtong
}

\section{THEME}

Many older neighborhood areas in Chinese cities keep their own specifics, but the living conditions there are growing worse. How to find an appropriate approach adapting to the local culture and features of the city and create an ideal, comfortable, graceful, safe, and convenient living environment in the older neighborhood areas is the purpose of our scheme.

\section{SITE SPECIFICATIONS}

The site of this project is one of the most densely populated areas in Shanghai, which is the largest city of China. The most typical dwelling here is the Lane, the integration of western rank housing and the Chinese traditional house. But now either the living situation or the environmental quality here is getting bad, with overly high density, sharing kitchens, and with little or no sanitation. The destruction and undesigned reconstruction have destroyed the networks and features of the city. There are also problems with the elderly and children within their activity lots. Many old customs and traditions of Lane living styles are gradually dying out.

\section{SCHEME BASIS}

1. The social foundation is based on the local lifestyle and customs due to Lane of Shanghai, as well as the population investigation.

2. The architectural design bases are the features and evolution of the Lane house, trying to create a kind of "Grade Separation Lane."

3. During the environmental design, Otis elevators provide the opportunity to set the public utilities on the first floor. The design method of Chinese traditional gardens is also used on the outdoor space design. Spaces can be placed in such an order: public activity space, public communication space, semi-private space (terrace), family living space, private family space.

\section{SCHEME EFFECT}

1. Higher density: FAR $54 \%, 26,000 \mathrm{~m}^{2} / \mathrm{ha}$

2. Providing every household reasonable living floor areas (both expanding family and nuclear family), satisfying the functional needs: physical (sunlight, through-ventilation, etc.), and psychical (safety, communication, opening to nature, keeping formal lifestyle, getting along with each other, etc.).

3. Evidently prompting the environmental quality (greenland, playing lots, etc.).

4. Satisfying the needs of community living (an elderly club and teahouse, a young people's club and library), providing sufficient public utilities (parking lots, stores, clinic, kindergarten, fastfood, etc.).

5. The scheme can be popularized as a helpful attempt; it may be put in practice either on a small or large scale.

\section{JURY COMMENTS}

This project takes on the block in a very direct way. It's not just a simple case of coming up with a unit type and then repeating them all over the place; in this scheme there clearly is a distinction between the housing that goes around the perimeter and that which is on the interior. It's not a traditional scheme, it's not an eclectic one, but it works with the Shanghai "type;" the language issues are handled in a very sophisticated way. Like all of the winning projects, it is not simply a housing scheme, but an urban block in which the major component is housing. 


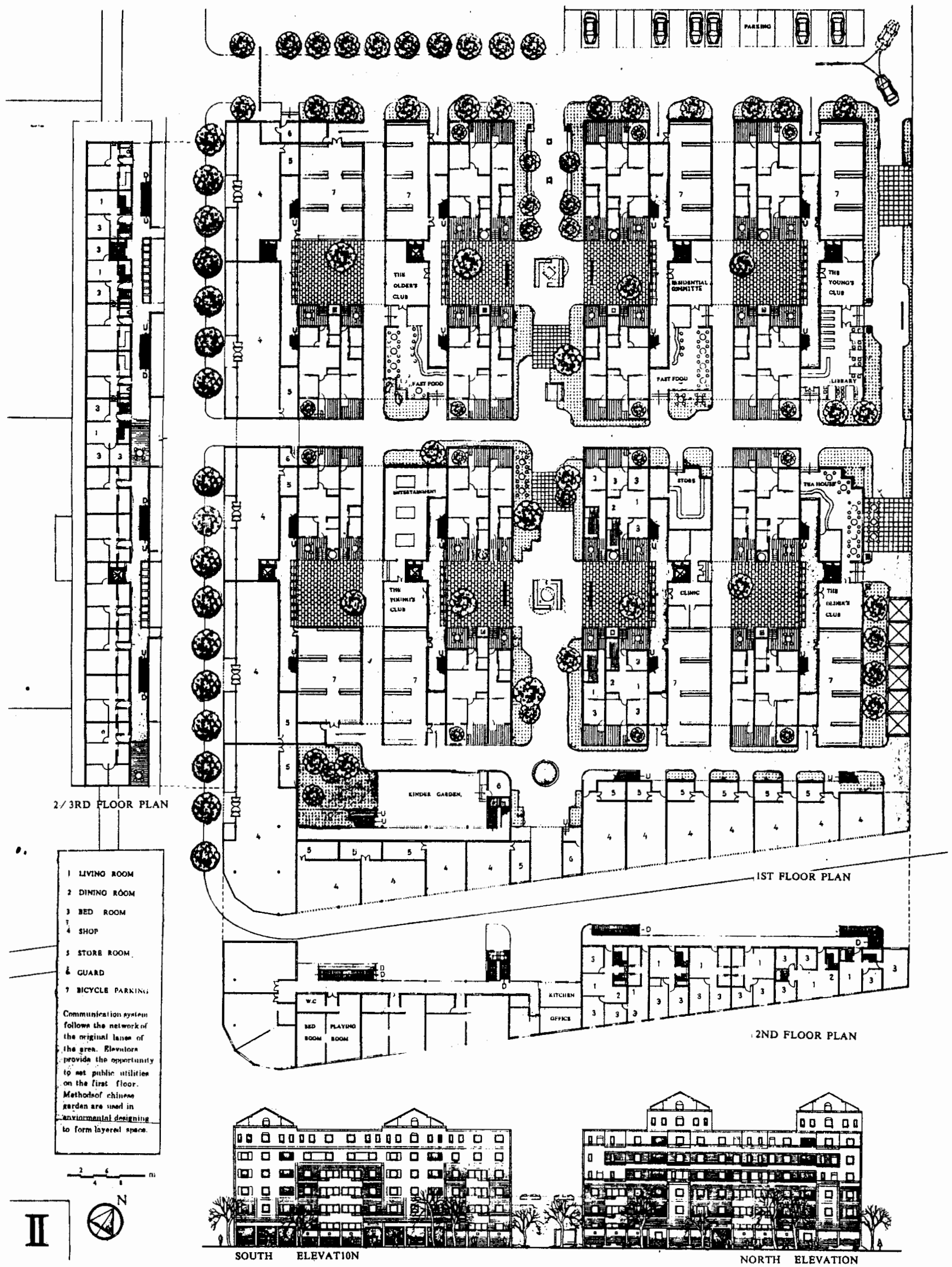

